\title{
Investigation of SSR characteristics of unified power flow controller
}

\author{
K.R. Padiyar*, Nagesh Prabhu \\ Department of Electrical Engineering, Indian Institute of Science, Bangalore 560012, India
}

\begin{abstract}
The unified power flow controller (UPFC) is the most versatile flexible ac transmission system (FACTS) controller which can be used to control active and reactive power flows in a transmission line in addition to the bus voltage. The active series compensation is provided by injecting series reactive voltage. The voltage at the two ports of UPFC are regulated by control of shunt current and series real voltage. It also has several operating control modes such as voltage and power regulation, line impedance compensation, etc. This paper presents the analysis and study of sub-synchronous resonance (SSR) characteristics of UPFC. The various combination of operating modes of shunt and series converters are considered for investigating their effect on SSR characteristics.

The analysis of SSR with UPFC is carried out based on frequency domain method, eigenvalue analysis and transient simulation. The frequency domain method considers D-Q model of UPFC for the computation of damping torque for quick check in determining torsional mode stability. The study is performed on IEEE First Benchmark Model (FBM).
\end{abstract}

Keywords: FACTS; Voltage source converter (VSC); UPFC; SSR; Damping torque

\section{Introduction}

Series compensation of long lines is an economic solution to the problem of enhancing power transfer and improving system stability. However, series compensated transmission lines connected to turbogenerators can result in SSR due to the negative damping introduced by the electrical network. This can cause self excitation due to torsional interaction and induction generator effect. The reduction of damping at torsional frequencies can also result in magnification of shaft torque oscillations caused by transient disturbances [1]. The hybrid series compensation consisting of a suitable combination of passive elements and active FACTS controller such as thyristor controlled series capacitor (TCSC) or static synchronous series compensator (SSSC) can be used to mitigate SSR.

The UPFC is the most versatile of FACTS controller capable of control of three system parameters; voltage, power

\footnotetext{
* Corresponding author. Tel.: +91 802293 2694; fax: +91 8023600444. E-mail address: krpyar@ee.iisc.ernet.in (K.R. Padiyar).
}

angle and transfer impedance. The schematic of the UPFC as a part of single machine infinite bus (SMIB) system (used in IEEE FBM) is shown in Fig. 1.

It consists of a shunt connected voltage source converter (VSC1) and a series connected voltage source converter (VSC2). VSC2 injects a series voltage while VSC1 is controlled to inject reactive current. The series and shunt branches of UPFC can generate/absorb reactive power independently and the two branches can exchange active power. The injection of series reactive voltage provides active series compensation while the injection of the shunt reactive current can be controlled to regulate the voltage at the bus where VSC1 is connected. The injection of series real voltage (in-phase with the line current) can be controlled to regulate the reactive power in the line or the voltage at the output port of the UPFC (The UPFC can be viewed as a two port device on a single phase basis) [2].

The capacitor voltage is regulated at the specified value by dc voltage controller to maintain power balance between shunt and series branches. 


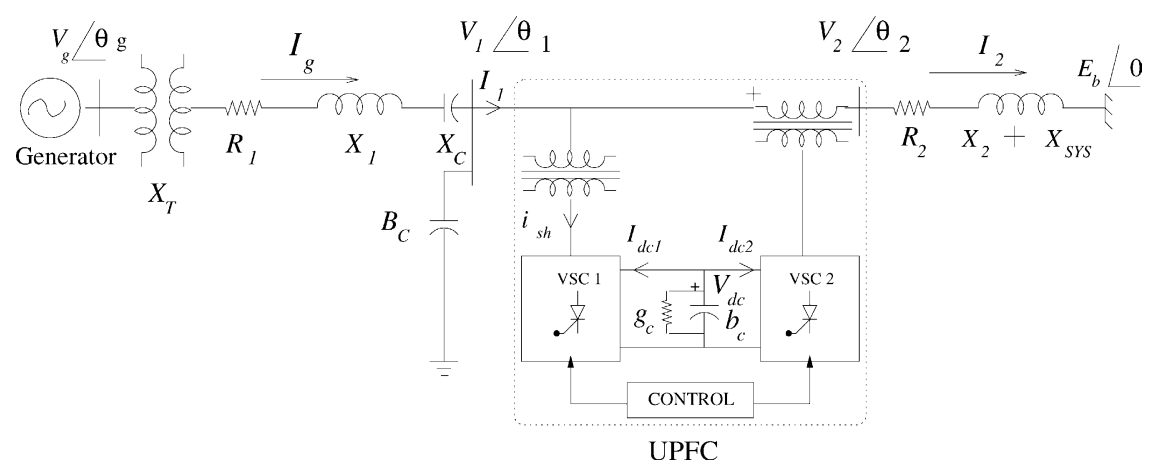

Fig. 1. Electrical system of IEEE FBM with UPFC.

The power balance can be expressed mathematically as

$\Re\left(\bar{V}_{1} \bar{I}_{1}^{*}-\bar{V}_{2} \bar{I}_{2}^{*}\right)-P_{\text {loss }}=0$

In this paper, the UPFC is realized by two three-level 12pulse voltage source converters (VSC). The IEEE FBM is considered for the analysis of SSR. The objective is to investigate the detailed SSR characteristics of UPFC at different operating modes and examine the role of an UPFC as a SSR countermeasure.

As there is no published work on the analysis of SSR with UPFC, it becomes essential to establish the validity of the models used. This is performed by using a detailed nonlinear three-phase system model (including the switching action within the VSC) for transient simulation which is also used to validate the D-Q models employed for the linear analysis. While eigenvalue analysis is accurate and gives comprehensive information about the system stability at an operating point, simpler frequency domain techniques such as damping torque analysis can be used for a fast determination of stability of torsional modes.

This paper is organized as follows. Section 2 describes the modelling of UPFC based on three-level VSCs and the associated controllers. Section 3 gives a brief account of the analytical methods used while Section 4 illustrates these using the example based on IEEE FBM. The conclusions are drawn in Section 5.

\section{Modelling of UPFC with three-level VSC}

In the power circuit of a UPFC, the converter is usually either a multi-pulse or a multilevel configuration. In UPFC, it is desirable to vary the magnitude of ac output voltage of the converter without changing the magnitude of the dc voltage. This can be possible by pulse width modulation (PWM) with two level topology which demands higher switching frequency and leads to increased losses. The three-level converter topology can achieve the goal by varying dead angle $\beta$ with fundamental switching frequency [3]. The converters that allow the variation of both magnitude and the phase angle of converter output voltage are classified as type- 1 converters [4]. Here a combination of multi-pulse and three-level config- uration [5] is considered. The three-level converter topology greatly reduces the harmonic distortion on the ac side [3,6-8].

Both the shunt and series branches of the UPFC consists of 12-pulse converter with three-level poles. The detailed threephase model of UPFC is developed by modelling the converter operation by switching functions [5].

\subsection{Mathematical model in $D$ - $Q$ frame of reference}

When switching functions are approximated by their fundamental frequency components neglecting harmonics, UPFC can be modelled by transforming the three-phase voltages and currents to D-Q variables using Kron's transformation [9]. The UPFC can be represented functionally as a two port device as shown in Fig. 2.

In Fig. $2, R_{\mathrm{sh}}, X_{\mathrm{sh}}$ and $R_{\mathrm{se}}, X_{\mathrm{se}}$ are the resistance and reactance of the interfacing transformer of shunt and series VSC, respectively. The magnitude control of shunt and series converter output voltages $V_{(\mathrm{sh})}^{i}$ and $V_{(\mathrm{se})}^{i}$ is achieved by modulating the conduction period affected by dead angle $\beta_{\mathrm{sh}}$ and $\beta_{\text {se }}$ of individual converters while dc voltage is maintained constant.

The shunt converter output voltage can be represented in $\mathrm{D}-\mathrm{Q}$ frame of reference as

$$
\begin{aligned}
& V_{(\mathrm{sh})}^{i}=\sqrt{V_{\mathrm{D}(\mathrm{sh})}^{2}+V_{\mathrm{Q}(\mathrm{sh})}^{2}} \\
& V_{\mathrm{D}(\mathrm{sh})}=k_{\mathrm{m} 1} V_{\mathrm{dc}} \sin \left(\theta_{1}+\alpha\right)
\end{aligned}
$$

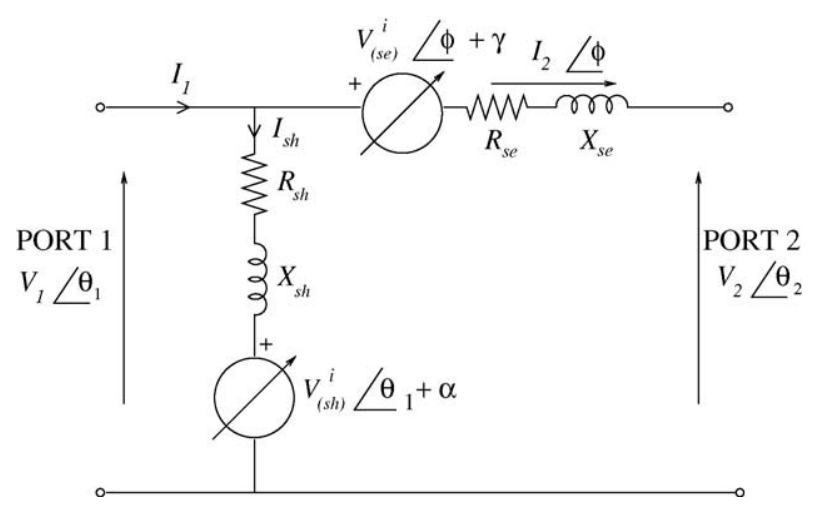

Fig. 2. UPFC as a two port FACTS controller. 
$V_{\mathrm{Q}(\mathrm{sh})}=k_{\mathrm{m} 1} V_{\mathrm{dc}} \cos \left(\theta_{1}+\alpha\right)$

where $k_{\mathrm{m} 1}=k \rho_{\mathrm{sh}} \cos \left(\beta_{s h}\right), k=\frac{2 \sqrt{6}}{\pi}$ for a 12-pulse converter and $\rho_{\mathrm{sh}}$ is the transformation ratio of the interfacing transformer $T_{1} . \alpha$ is the angle by which the fundamental component of shunt converter output voltage leads the port-1 voltage $V_{1}$.

The series converter output voltage $V_{(\mathrm{se})}^{i}$ can also be represented in D-Q frame of reference in a similar fashion as given in Eqs. (2)-(4) except that $\alpha$ is replaced by $\gamma$ and $k_{\mathrm{m} 1}$ by $k_{\mathrm{m} 2}$, where $k_{\mathrm{m} 2}=k \rho_{\mathrm{se}} \cos \left(\beta_{\mathrm{se}}\right), \rho_{\mathrm{se}}$ is the transformation ratio of the interfacing transformer $\mathrm{T}_{2}, \gamma$ is the angle by which the fundamental component of series converter output voltage leads the port- 2 current $I_{2}$.

The dc side capacitor is described by the dynamical equation as

$\frac{\mathrm{d} V_{\mathrm{dc}}}{\mathrm{d} t}=-\frac{g_{\mathrm{c}} \omega_{\mathrm{B}}}{b_{\mathrm{c}}}-I_{\mathrm{dc} 1} \frac{\omega_{\mathrm{B}}}{b_{\mathrm{c}}}-I_{\mathrm{dc} 2} \frac{\omega_{\mathrm{B}}}{b_{\mathrm{c}}}$

where $\quad I_{\mathrm{dc} 1}=-\left[k_{\mathrm{m} 1} \sin \left(\theta_{1}+\alpha\right) I_{\mathrm{shD}}+k_{\mathrm{m} 1} \cos (\theta 1+\alpha) I_{\mathrm{shQ}}\right]$; $I_{\mathrm{shD}}$ and $I_{\mathrm{shQ}}$ are D-Q components of shunt converter current; $I_{\mathrm{dc} 2}=-\left[k_{\mathrm{m} 2} \sin (\phi+\gamma) I_{2 \mathrm{D}}+\cos (\phi+\gamma) I_{2 \mathrm{Q}}\right]$.

\subsection{Shunt current control}

The real current drawn by the shunt VSC is controlled by phase angle $\alpha$ and reactive current by modulating the converter output voltage magnitude as a function of $\beta_{\mathrm{sh}}$. In [2], the dynamical equations of the shunt current control are dealt with in detail. Fig. 3 shows the schematic representation of type-1 controller for shunt current control. The reactive current reference of shunt converter can be kept constant or regulated to maintain port-1 voltage magnitude at the specified value.

In Fig. 3, real and reactive currents are defined as

$$
\begin{aligned}
& I_{\mathrm{P}(\mathrm{sh})}=I_{\mathrm{shD}} \sin \left(\theta_{1}\right)+I_{\mathrm{shQ}} \cos \left(\theta_{1}\right) \\
& I_{\mathrm{R}(\mathrm{sh})}=-I_{\mathrm{shD}} \cos \left(\theta_{1}\right)+I_{\mathrm{shQ}} \sin \left(\theta_{1}\right)
\end{aligned}
$$

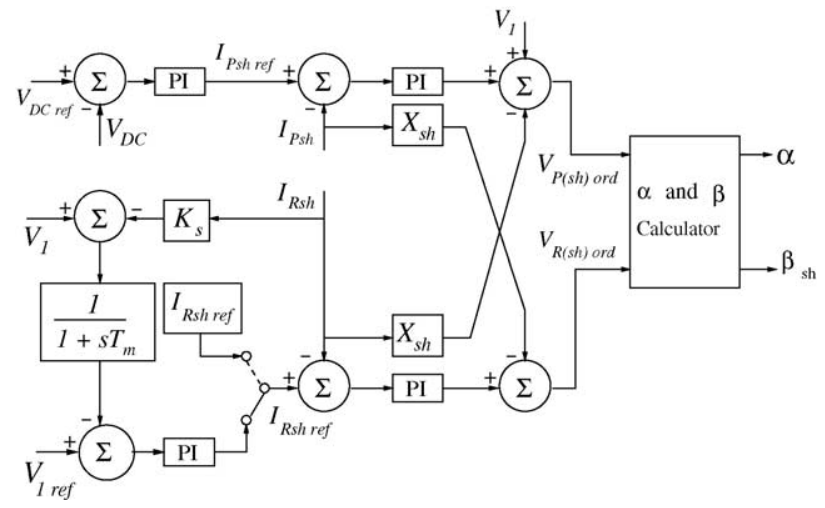

Fig. 3. Shunt current controller. and $\alpha$ and $\beta_{\mathrm{sh}}$ are calculated as

$\alpha=\tan ^{-1}\left[\frac{V_{\mathrm{R}(\mathrm{sh})(\mathrm{ord})}}{V_{\mathrm{P}(\mathrm{sh})(\mathrm{ord})}}\right]$

$\beta_{\mathrm{sh}}=\cos ^{-1}\left[\frac{\sqrt{V_{\mathrm{P}(\mathrm{sh})(\mathrm{ord})}^{2}+V_{\mathrm{R}(\mathrm{sh})(\mathrm{ord})}^{2}}}{\kappa \rho_{\mathrm{sh}} V_{\mathrm{dc}}}\right]$

Eqs. (6) and (7), result in positive values when shunt VSC is drawing real current and inductive reactive current.

\subsection{Series voltage control}

The type-1 controller structure for series converter is shown in Fig. 4.

The independent injection of real and reactive voltage give rise to two operating combinations of series converter as described below.

(1) Constant reactive voltage and port-2 voltage control.

(2) Constant reactive voltage and constant resistance emulation.

The active power can be regulated and/or modulated by controlling series reactive voltage reference $[10,11]$. The reactive power in the line can be regulated by controlling series real voltage injection and this is equivalent to port- 2 voltage control [2].

The voltage at port- 2 of the UPFC is algebraically related to that at port-1 and the voltage injected by series VSC (for simplicity the series transformer reactance is clubbed with the line impedance). The voltage relation is given by

$V_{2}=\left\{\begin{array}{l}\sqrt{\left(V_{2 \mathrm{D}}\right)^{2}+\left(V_{2 \mathrm{Q}}\right)^{2}} \\ \sqrt{\left(V_{1 \mathrm{D}}-V_{\mathrm{D}(\mathrm{se})}\right)^{2}+\left(V_{1 \mathrm{Q}}-V_{\mathrm{Q}(\mathrm{se})}\right)^{2}} \\ \sqrt{\left(V_{\mathrm{P} 1}-V_{\mathrm{P}(\mathrm{se})}\right)^{2}+\left(V_{\mathrm{R} 1}-V_{\mathrm{R}(\mathrm{se})}\right)^{2}}\end{array}\right.$

where $V_{\mathrm{P} 1}$ and $V_{\mathrm{R} 1}$ are the in-phase and quadrature components of port-1 voltage $V_{1}$ with respect to port-2 current and

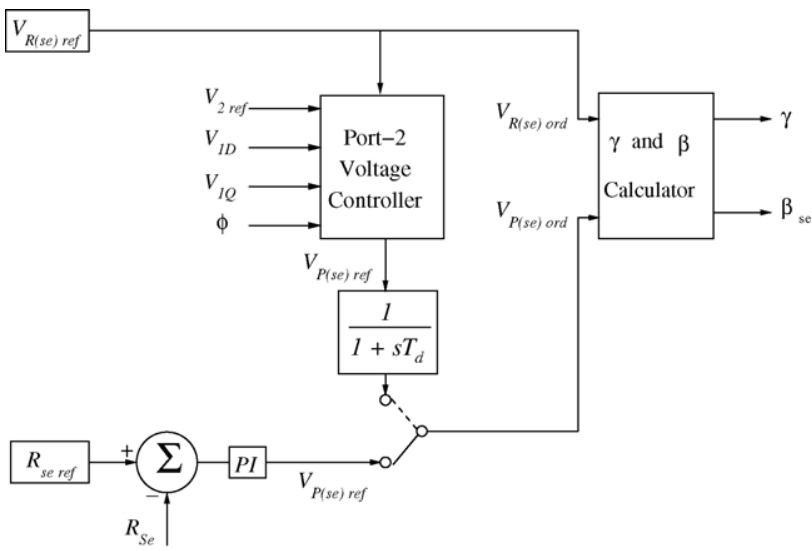

Fig. 4. Series voltage controller. 
Table 1

Operating modes for shunt and series VSC

\begin{tabular}{llll}
\hline Case & Shunt converter & Series converter & \\
\cline { 3 - 4 } & & Controller 1 & Controller 2 \\
\hline 1 & Port-1 voltage & Reactive voltage & Port-2 voltage \\
2 & Port-1 voltage & Reactive voltage & Resistance emulation \\
3 & Reactive current & Reactive voltage & Port-2 voltage \\
4 & Reactive current & Reactive voltage & Resistance emulation \\
\hline
\end{tabular}

similarly $V_{\mathrm{P}(\mathrm{se})}$ and $V_{\mathrm{R}(\mathrm{se})}$ are the in-phase and quadrature components of $V_{(\mathrm{se})}^{i}$ with respect to port-2 current and are expressed as

$$
\begin{aligned}
& V_{\mathrm{P}(\mathrm{se})}=V_{\mathrm{D}(\mathrm{se})} \sin (\phi)+V_{\mathrm{Q}(\mathrm{se})} \cos (\phi) \\
& V_{\mathrm{R}(\mathrm{se})}=V_{\mathrm{D}(\mathrm{se})} \cos (\phi)-V_{\mathrm{Q}(\mathrm{se})} \sin (\phi)
\end{aligned}
$$

In Eq. (10), we can compute $V_{\mathrm{P} 1}$ and $V_{\mathrm{R} 1}$ from $V_{1 \mathrm{D}}, V_{1 \mathrm{Q}}$ and $\phi$ by assuming $V_{\mathrm{R}(\mathrm{se})}=V_{\mathrm{R} \text { (se)ref. We can easily calculate }}$ real voltage $V_{\mathrm{P}(\mathrm{se})}$ to be injected to obtain desired magnitude of $V_{2}$ (see Fig. 4). There are two solutions of $V_{\mathrm{P}(\mathrm{se})}$; the solution which has a lower magnitude is chosen.

In Fig. $4, \gamma$ and $\beta_{\text {se }}$ are calculated as

$\gamma=\tan ^{-1}\left[\frac{V_{\mathrm{R}(\mathrm{se})(\text { ord })}}{V_{\mathrm{P}(\mathrm{se})(\mathrm{ord})}}\right]$

$\beta_{\mathrm{se}}=\cos ^{-1}\left[\frac{\sqrt{V_{\mathrm{P}(\mathrm{se})(\text { ord })}^{2}}+\sqrt{V_{\mathrm{R}(\mathrm{se})(\text { ord })}^{2}}}{k \rho_{\mathrm{se}} V_{\mathrm{dc}}}\right]$

The various operating modes of UPFC considered are summarized in Table 1. These are not exhaustive as the reactive voltage reference for the controller can be set by an outer controller regulating power flow in the line.

\section{Analysis of SSR}

The analysis of SSR with UPFC is carried out based on damping torque analysis, eigenvalue analysis and transient simulation.

\subsection{Damping torque analysis}

Damping torque analysis is a frequency domain method which can be used to screen the system conditions that give rise to potential SSR problems. The significance of this approach is that it enables the planners to decide upon a suitable countermeasure for the mitigation of the detrimental effects of SSR. Damping torque method gives a quick check to determine the torsional mode stability. The system is assumed to be stable if the net damping torque at any of the torsional mode frequency is positive [12].

The interaction between the electrical and mechanical system can be represented by the block diagram shown in Fig. 5 .

At any given oscillation frequency of the generator rotor, the component of electrical torque $\left(\Delta T_{\mathrm{e}}\right)$ in phase with

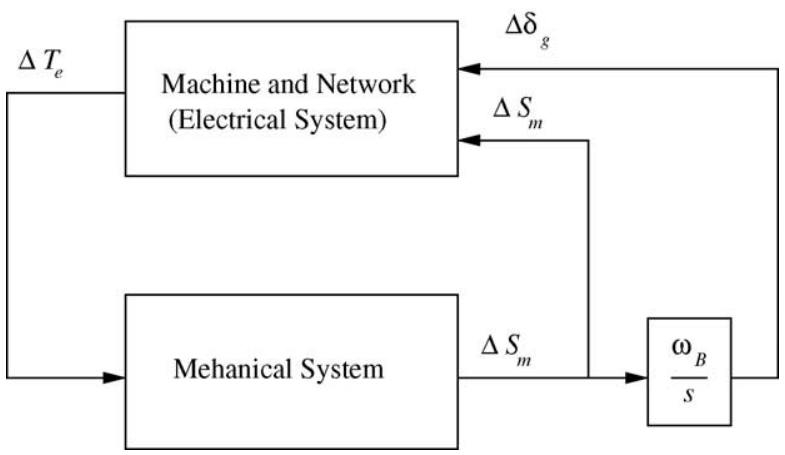

Fig. 5. Interaction between mechanical and electrical system.

the rotor speed $\left(\Delta S_{\mathrm{m}}\right)$ is termed as damping torque. The expression for damping torque coefficient $\left(T_{\mathrm{de}}\right)$ by the external transmission network is derived in [1]. Although it is possible to consider the detailed model of the generator in the computation of damping torque, it is convenient to model the generator with classical model (constant voltage source $\left(E^{\prime}\right)$ behind a transient reactance $\left.\left(X^{\prime}\right)\right)$ if the objective is to study mainly the torsional interactions. This assumption is equivalent to neglecting induction generator effect (IGE) and does not have a significant effect on the prediction of torsional mode stability. The stability of mode 0 (corresponding to low frequency oscillations) is obviously dependent on the generator model considered. Here, detailed generator model is required [13].

The electrical torque $\left(\Delta T_{\mathrm{e}}\right)$ as a function of the change in per unit rotor speed $\left(\Delta S_{\mathrm{m}}\right)$ can be derived from the knowledge of the impedance functions. At the generator internal bus the following equation applies.

$\left[\begin{array}{c}\Delta I_{\mathrm{gD}}(j \omega) \\ \Delta I_{\mathrm{gQ}}(j \omega)\end{array}\right]=\left[\begin{array}{l}Y_{\mathrm{DD}}(j \omega) Y_{\mathrm{DQ}}(j \omega) \\ Y_{\mathrm{QD}}(j \omega) Y_{\mathrm{QQ}}(j \omega)\end{array}\right]\left[\begin{array}{l}\Delta E_{\mathrm{gD}}(j \omega) \\ \Delta E_{\mathrm{gQ}}(j \omega)\end{array}\right]$

The expression for the damping torque can be written as

$T_{\mathrm{de}}(\omega)=\Re\left[\frac{\Delta T_{\mathrm{e}}(j \omega)}{\Delta S_{\mathrm{m}}(j \omega)}\right]$

and can be expressed as [1]

$T_{\mathrm{de}}(\omega)=\Re\left[Y_{\mathrm{QD}}(j \omega) \frac{\omega_{0}}{j \omega}+Y_{\mathrm{QQ}}(j \omega)\right]\left(E^{\prime}\right)^{2}$

The derivation of $Y_{\mathrm{DD}}, Y_{\mathrm{DQ}}, Y_{\mathrm{QD}}$ and $Y_{\mathrm{QQ}}$ as functions of the frequency is given in Appendix A. When the mechanical damping is zero, the instability of $i$ th torsional mode frequency $\omega_{i}$ is determined from the criterion $T_{\mathrm{de}}\left(\omega_{i}\right)<0$ and the decrement factor $\sigma_{i}$ can be approximately expressed as $[1,12]$

$\sigma_{i}=-\frac{T_{\mathrm{de}}\left(\omega_{i}\right)}{4 H_{\mathrm{m} i}}$

where $H_{\mathrm{m} i}$ is the modal inertia for the $i$ th mode. 


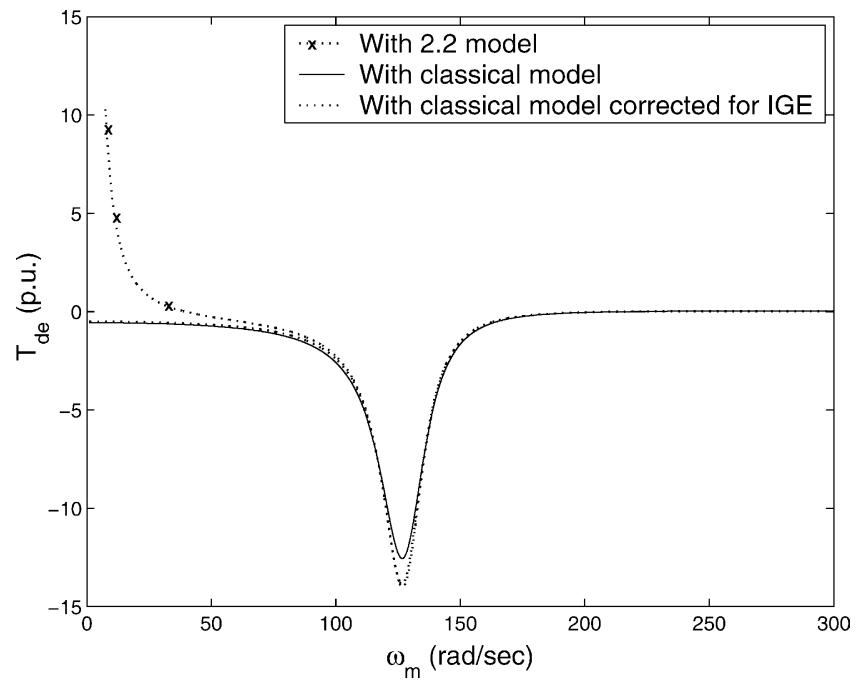

Fig. 6. Comparison of damping torques with detailed and classical model of generator.

\subsubsection{Comparison of damping torque with classical and detailed model of generator}

The effect of IGE due to detailed (2.2) generator model [9] on torsional mode stability is considered for the system of Fig. 1 with $(X / R)$ ratio 12.5 for the case without UPFC. The (2.2) model represents four rotor windings, two each on $\mathrm{D}$ and $\mathrm{Q}$ axes, including the field winding. The series capacitor compensation is set to $60 \%$ of the transmission line reactance. The variation of damping torque with the detailed and classical model of generator (which neglects IGE) as a function of frequency is shown in Fig. 6.

As mentioned earlier, the damping torque at low frequencies $\left(\omega_{\mathrm{m}}<50\right)$ is affected by the generator model. However, the peak negative damping torque occur at the same resonant frequency $\left(\omega_{\mathrm{m}}=126.82\right)$ for both models. The peak value of the negative damping torque is slightly higher for the detailed generator model due to the negative resistance introduced by IGE. The correction for IGE is possible by adding a small negative resistance to the total resistance of the electrical system. This value of negative resistance can be accurately computed by the impedance offered by the generator.

The phase impedance (encountered by armature currents) at a frequency $\omega$ can be computed as [1]

$$
\begin{aligned}
Z_{\mathrm{g}}(j \omega)= & \left(R_{\mathrm{g}}(j \omega)+j X_{\mathrm{g}}(j \omega)\right) \\
= & \frac{1}{2}\left\{\left[Z_{\mathrm{gDD}}\left(j\left(\omega-\omega_{0}\right)\right)+Z_{\mathrm{gQQ}}\left(j\left(\omega-\omega_{0}\right)\right)\right]\right. \\
& \left.+j\left[Z_{\mathrm{gDQ}}\left(j\left(\omega-\omega_{0}\right)\right)-Z_{\mathrm{gQD}}\left(j\left(\omega-\omega_{0}\right)\right)\right]\right\}
\end{aligned}
$$

where

$$
\begin{aligned}
& Z_{\mathrm{gDD}}(\mathrm{s})=\frac{s}{\omega_{\mathrm{B}}} X_{\mathrm{d}}(\mathrm{s}), \quad Z_{\mathrm{gDQ}}(\mathrm{s})=\frac{\omega_{0}}{\omega_{\mathrm{B}}} X_{\mathrm{q}}(\mathrm{s}) \\
& Z_{\mathrm{gDQ}}(\mathrm{s})=-\frac{\omega_{0}}{\omega_{\mathrm{B}}} X_{\mathrm{d}}(\mathrm{s}), \quad Z_{\mathrm{gQQ}}(\mathrm{s})=\frac{s}{\omega_{\mathrm{B}}} X_{\mathrm{q}}(\mathrm{s})
\end{aligned}
$$
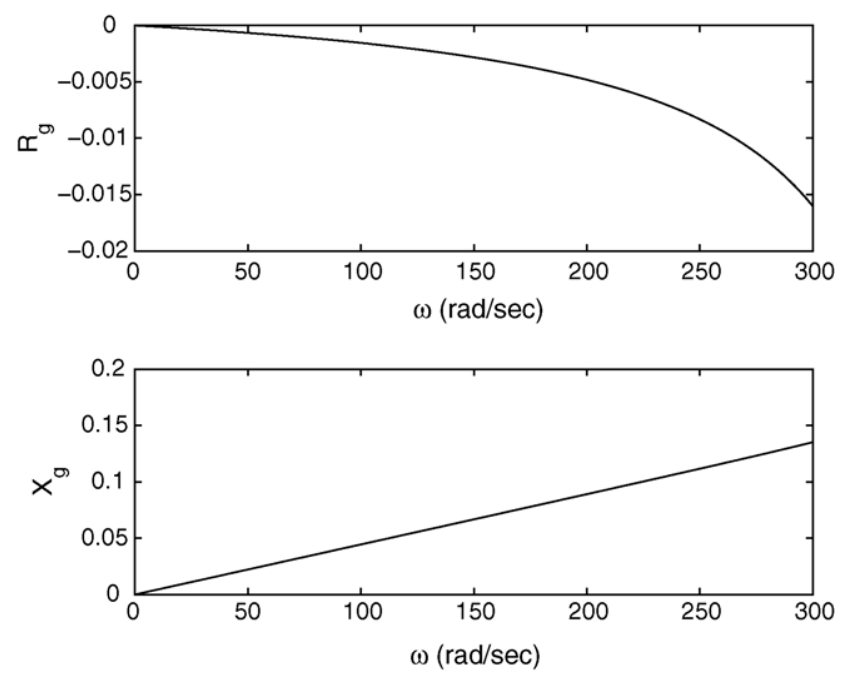

Fig. 7. Resistance and reactance of 2.2 model of generator.

$X_{\mathrm{d}}(\mathrm{s})=\frac{x_{\mathrm{d}}\left(1+s T_{\mathrm{d}}^{\prime}\right)\left(1+S{T_{\mathrm{d}}^{\prime \prime}}^{\prime}\right)}{\left(1+s T_{\mathrm{d} 0}^{\prime}\right)\left(1+S{T_{\mathrm{d} 0}^{\prime}}^{\prime}\right)}$

$X_{\mathrm{q}}(\mathrm{s})=\frac{x_{\mathrm{q}}\left(1+s T_{\mathrm{q}}^{\prime}\right)\left(1+S{T_{\mathrm{q}}^{\prime \prime}}^{\prime}\right)}{\left(1+s T_{\mathrm{q} 0}^{\prime}\right)\left(1+S{T_{\mathrm{q} 0}^{\prime \prime}}^{\prime}\right)}$

The resistance $R_{\mathrm{g}}$ and reactance $X_{\mathrm{g}}$ of generator are shown in Fig. 7 as functions of frequency $(\omega)$. It is seen that, $R_{\mathrm{g}}$ is negative for $\omega<\omega_{0}$. Also, $X_{\mathrm{g}}$ is a linear function of $\omega$, based on constant p.u. reactance $\left(X^{\prime}\right)$.

For considering the effect of IGE in classical model, the negative resistance calculated at the critical frequency is added to the transmission line resistance as a correction factor. At the operating point considered, the torsional mode- 2 is unstable and the negative resistance (due to IGE) is -0.0083 . The damping torque analysis with classical model after correcting for IGE (see Fig. 6) shows that, the peak negative damping torque is nearly same as that obtained with 2.2 generator model. Hence, the classical model is adequate for fast prediction of the torsional mode stability as the effect of IGE is not very significant in practical cases.

\subsection{Eigenvalue analysis}

In this analysis, generator model (2.2) [9] is considered. The electromechanical system consists the multi-mass mechanical system, the generator, the excitation system, power system stabilizer (PSS), torsional filter and the transmission line with UPFC. The UPFC Eqs. (2)-(14) along with the equations representing electromechanical system [1,9] (in D$\mathrm{Q}$ variables), are linearized at the operating point and eigenvalues of system matrix are computed. The stability of the system is determined by the location of the eigenvalues of system matrix. The system is stable if the eigenvalues have negative real parts. 


\subsection{Transient simulation}

The eigenvalue analysis uses equations in D-Q variables where the switching functions are approximated by their fundamental frequency components (converter switchings are neglected). To validate the results obtained from damping torque and eigenvalue analysis, the transient simulation should be carried out using detailed nonlinear three-phase model of UPFC which considers the switching in the threephase converters. Hence, the UPFC is modelled by two threelevel 12-pulse converters by generating switching functions. The transient simulation of the combined nonlinear system with detailed three-phase model of UPFC is carried out using MATLAB-SIMULINK [14].

\section{A case study}

The system considered is adapted from IEEE FBM [15]. The electrical system is represented schematically in Fig. 1, which consists of a generator and series compensated long transmission line with UPFC. The shunt capacitor $\left(B_{\mathrm{c}}\right)$ and VSC1 is located at the electrical center of the transmission line (considering series injected reactive voltage). The generator and mechanical system data are taken from $[1,15]$.

The modelling aspects of the electromechanical system comprising the generator, the multi-mass mechanical system, the excitation system, power system stabilizer (PSS), torsional filter and the transmission line containing the series capacitor are given in detail in [1,9].

The analysis is carried out on the IEEE FBM based on the following initial operating condition and assumptions.

(1) The generator delivers 0.9 p.u. power to the transmission system.

(2) The input mechanical power to the turbine is assumed constant.

(3) The total series compensation level is set at 0.6 p.u.

(4) The turbine-generator mechanical damping is neglected for damping torque analysis.

\subsection{Results of damping torque analysis}

The damping torque method involves less computational burden and is a convenient tool for analyzing the SSR characteristics of the electrical network. Damping torque analysis can be used to predict the potential SSR problems under various system operating conditions.

\subsubsection{Damping torque analysis with $V_{P(s e)}=0.0$}

The total series compensation of $60 \%$ is met by hybrid compensation wherein $40 \%$ of compensation is met by fixed capacitor and the remaining 20\% is realized by injected series reactive voltage by UPFC, at the operating point. The initial operating value of $V_{\mathrm{P}(\mathrm{se})}=0.0$. In all cases, the shunt reactive current is adjusted to a value to obtain the magnitude of port-

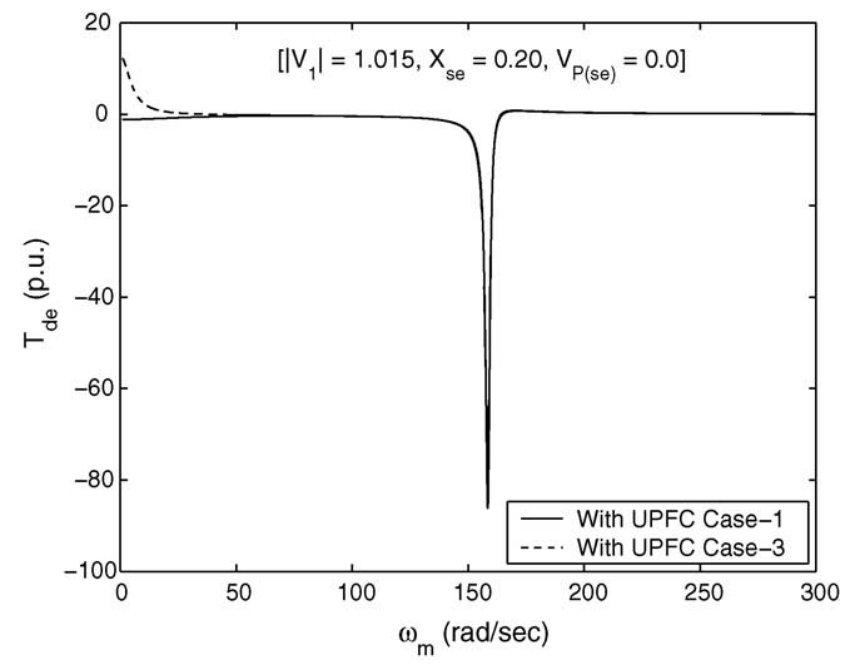

Fig. 8. Plot of damping torque with frequency for cases 1 and 3.

1 voltage of 1.015 p.u. In steady state, the operating value of $I_{\text {Rsh }}=-0.0856$.

The variation of damping torque with frequency for cases 1-4 (as given in Table 1) are shown in Figs. 8 and 9, respectively.

It is to be noted that in all the cases, maximum negative damping occurs at around $160 \mathrm{rad} / \mathrm{s}$ which matches with torsional mode 3 of IEEE FBM and adverse torsional interactions are expected.

It is to be noted that peak negative damping with cases 1 and 3 is nearly about -86 p.u. associated with a sharp dip. With cases 2 and 4 the peak negative damping is about -37.5 p.u. indicating that resistance emulation gives better SSR characteristics compared to port- 2 voltage control. It is observed that, with constant reactive current control mode of shunt converter (cases 3 and 4), the peak negative damping torque is marginally reduced compared to constant voltage (at port-1) control.

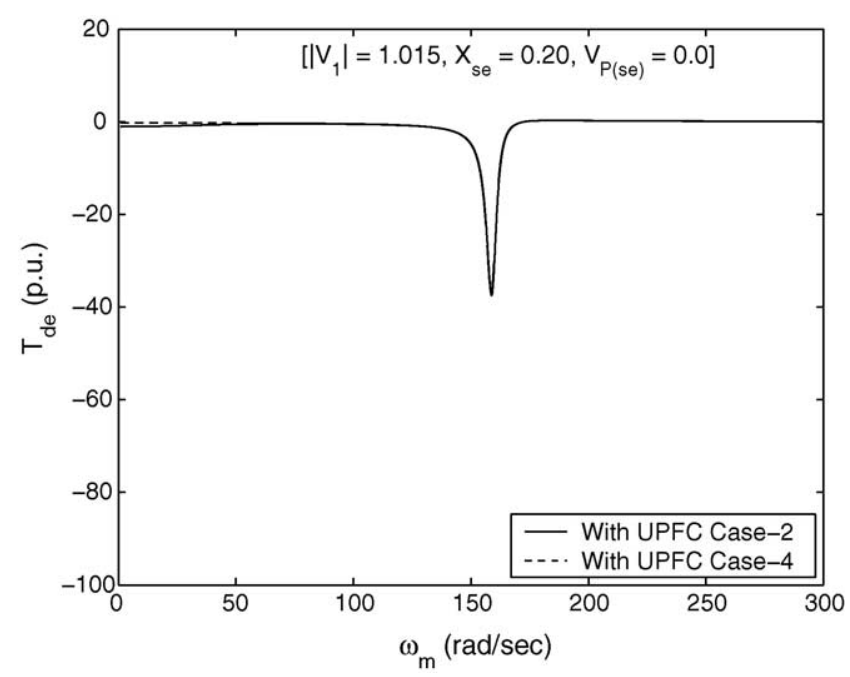

Fig. 9. Plot of damping torque with frequency for cases 2 and 4. 


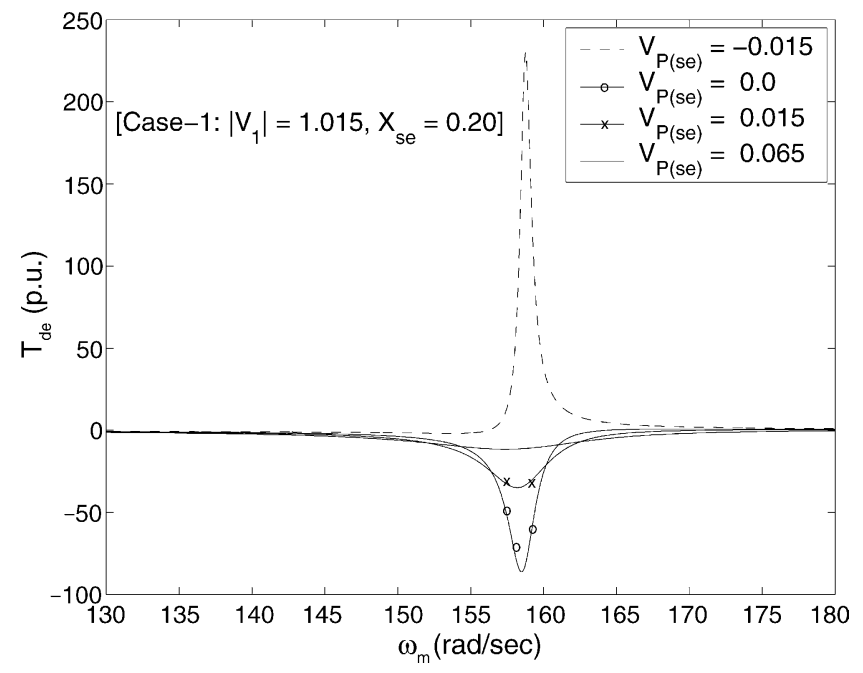

Fig. 10. Sensitivity of damping torque for variation in $V_{\mathrm{P}(\mathrm{se})}$.

\subsubsection{Sensitivity of damping torque for series real voltage $\left(V_{P(s e)}\right)$ injection}

The peak negative damping associated with various operating cases depends also on the magnitude of series real voltage injection. The variation of damping torque with frequency for case 1 is shown in Fig. 10. Referring to Fig. 10, peak negative damping is reduced substantially with positive real voltage injection $V_{\mathrm{P}(\mathrm{se})}=0.065$, which emulates positive resistance in series with the line. However, negative real voltage injection $V_{\mathrm{P}(\mathrm{se})}=-0.015$ (which emulates negative resistance) can destabilize the network mode. The damping torque remains practically unchanged in the regions $0-130$ and $180-300 \mathrm{rad} / \mathrm{s}$ (not shown in Fig. 10).

\subsubsection{Sensitivity of damping torque for series reactive voltage $\left(V_{R(s e)}\right)$ injection}

The effect of increase in series injected capacitive reactive voltage (hence $X_{\mathrm{se}}$ ) and keeping total compensation at $60 \%$, is shown in Fig. 11. It is observed that, increased injection of capacitive reactive voltage causes significant increase in the frequency at which resonance occurs. While the peak negative damping increases with increase in $V_{\mathrm{R}(\mathrm{se})}$ for case 1 (constant voltage control), it decreases for case 3 (constant reactive current control). However, these results may not apply in general. The damping torque remains practically unchanged in the regions $0-120$ and $180-300 \mathrm{rad} / \mathrm{s}$.

\subsubsection{Sensitivity of damping torque for shunt reactive current $\left(I_{\text {Rsh }}\right)$ injection}

The effect of injection of shunt reactive current is shown in Fig. 12. The injection of increased capacitive reactive current by shunt converter increases the resonance frequency marginally and decreases the damping of torsional modes. As mentioned earlier, the results for case 2 indicates better damping with resistance emulation. The damping torque remains practically unchanged in the regions $0-130$ and $180-300 \mathrm{rad} / \mathrm{s}$.
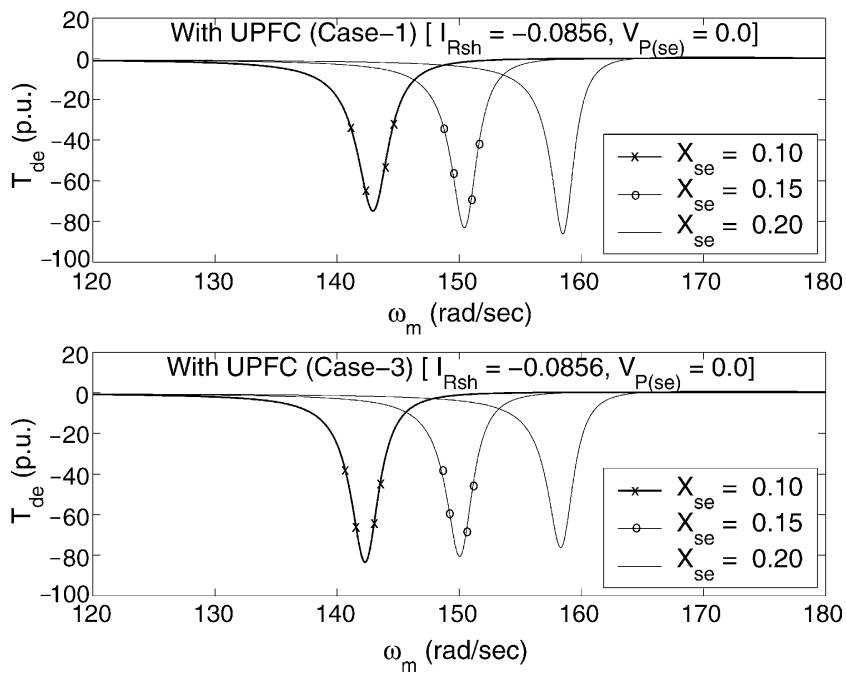

Fig. 11. Sensitivity of damping torque for variation in $X_{(\mathrm{se})}$.

\subsection{Eigenvalue analysis}

In this analysis, the turbine-generator mechanical damping is considered and generator is modelled with 2.2 model (as indicated in Section 3.2). Here the operating value of series injected real voltage is set at $V_{\mathrm{P}(\mathrm{se})}=0.0$, $X_{\mathrm{se}}=\left(-V_{\mathrm{R}(\mathrm{se})} / I_{2}\right)=0.2, \quad X_{\mathrm{c}}=0.4$ and port-1 voltage at $\left|V_{1}\right|=1.015$ p.u. as in Section 4.1.1. The eigenvalues of system matrix are computed for cases 1, 3,2 and 4 are given in Tables 2 and 3. Comparing the eigenvalue results of Tables 2 and 3, it is to be noted that, the mode of control of shunt VSC has no significant effect on the torsional modes, whereas damping of mode 0 has improved with port- 1 voltage control. The latter fact is not in agreement with the results of damping torque analysis (shown in Figs. 6 and 7) which assume classical model of the generator. However, eigenvalues
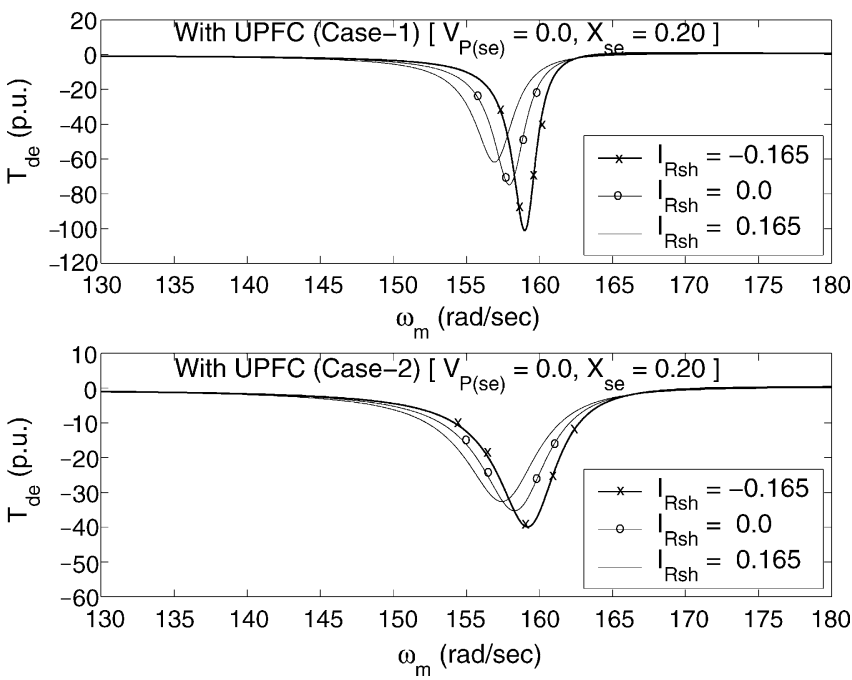

Fig. 12. Sensitivity of damping torque for variation in $I_{\text {Rsh }}$. 
Table 2

Eigenvalues of the detailed system $\left(V_{\mathrm{P}(\mathrm{se})}=0\right)$

\begin{tabular}{lll}
\hline Torsional mode & \multicolumn{2}{l}{ Eigenvalue } \\
\cline { 2 - 3 } & Case 1 & Case 3 \\
\hline 0 & $-1.5903 \pm j 8.4618$ & $-1.1294 \pm j 7.7959$ \\
1 & $-0.2072 \pm j 99.1160$ & $-0.2109 \pm j 99.1210$ \\
2 & $-0.0699 \pm j 127.0400$ & $-0.0699 \pm j 127.0400$ \\
3 & $1.5185 \pm j 159.5400$ & $1.4113 \pm j 159.4800$ \\
4 & $-0.3914 \pm j 202.7900$ & $-0.3897 \pm j 202.8000$ \\
5 & $-1.8504 \pm j 298.1700$ & $-1.8504 \pm j 298.1700$ \\
Network mode & $-2.1188 \pm j 160.1800$ & $-2.1214 \pm j 160.1100$ \\
Network mode & $-1.5286 \pm j 560.4900$ & $-1.6869 \pm j 560.4400$
\end{tabular}

Table 3

Eigenvalues of the detailed system $\left(V_{\mathrm{P}(\mathrm{se})}=0\right)$

\begin{tabular}{lll}
\hline Torsional mode & \multicolumn{2}{l}{ Eigenvalue } \\
\cline { 2 - 3 } & Case 2 & Case 4 \\
\hline 0 & $-1.6923 \pm j 8.4382$ & $-1.4423 \pm j 8.2749$ \\
1 & $-0.1993 \pm j 99.1070$ & $-0.2055 \pm j 99.1120$ \\
2 & $-0.0688 \pm j 127.0400$ & $-0.0690 \pm j 127.0400$ \\
3 & $0.8427 \pm j 159.9300$ & $0.8045 \pm j 159.8800$ \\
4 & $-0.3850 \pm j 202.7900$ & $-0.3840 \pm j 202.8000$ \\
5 & $-1.8504 \pm j 298.1700$ & $-1.8504 \pm j 298.1700$ \\
Network mode & $-2.7931 \pm j 160.0700$ & $-2.7657 \pm j 159.9900$ \\
Network mode & $-1.3266 \pm j 560.1900$ & $-1.4757 \pm j 560.1500$ \\
\hline
\end{tabular}

corresponding to mode zero with classical model of the generator are (i) $0.0221 \pm j 7.8242$ and (ii) $-0.4191 \pm j 7.4542$ for cases 1 and 3, respectively. Thus, the modelling of the generator affects the stability of mode zero. The negative damping associated with mode 3 is maximum with case 1 and is in agreement with the conclusions of damping torque analysis. Table 4 gives the eigenvalues of the combined system when series real voltage is set to 0.065 p.u. and the controller emulates (positive) resistance in series with the line. All the torsional modes are stable in this case.

\subsection{Transient simulation}

The transient simulation of the combined system with detailed three-phase model of UPFC has been carried out for case 1 using MATLAB-SIMULINK [14]. The simulation results for case 1 with $V_{\mathrm{P}(\mathrm{se})}=0$ and 0.065 for a $10 \%$ decrease in input mechanical torque applied at $0.5 \mathrm{~s}$ and removed at

Table 4

Eigenvalues of the detailed system $\left(V_{\mathrm{P}(\mathrm{se})}=0.065\right)$

\begin{tabular}{lll}
\hline Torsional mode & \multicolumn{2}{l}{ Eigenvalue } \\
\cline { 2 - 3 } & Case 1 & Case 2 \\
\hline 0 & $-1.5799 \pm j 8.3975$ & $-1.5863 \pm j 8.121$ \\
1 & $-0.1898 \pm j 99.0480$ & $-0.1749 \pm j 99.0390$ \\
2 & $-0.0661 \pm j 127.0300$ & $-0.0621 \pm j 127.0300$ \\
3 & $-0.2198 \pm j 160.3000$ & $-0.3869 \pm j 202.8100$ \\
4 & $-0.3719 \pm j 202.8100$ & $-0.3669 \pm j 202.8100$ \\
5 & $-1.8504 \pm j 298.1700$ & $-1.8504 \pm j 298.1700$ \\
Network mode & $-6.0148 \pm j 158.4500$ & $-11.9020 \pm j 159.9200$ \\
Network mode & $-5.7150 \pm j 543.9000$ & $-16.8880 \pm j 545.2700$ \\
\hline
\end{tabular}
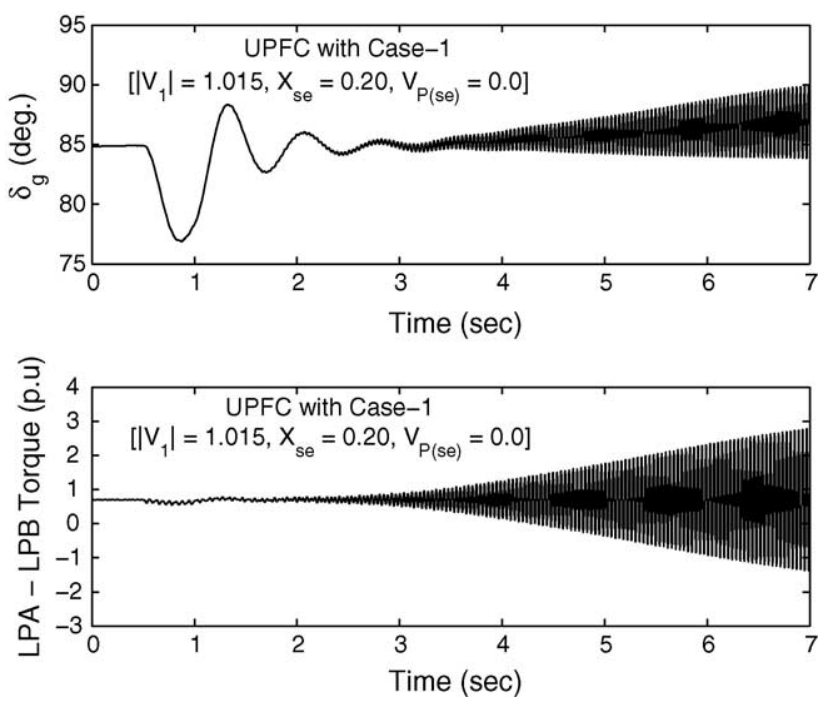

Fig. 13. Simulatiion with three-phase model of UPFC for step change in $T_{\mathrm{m}}$.

$1 \mathrm{~s}$ are shown in Figs. 13 and 14, respectively. The results are in agreement with what is predicted by eigenvalue analysis.

A large disturbance is initiated at $0.5 \mathrm{~s}$ in the form of three-phase fault at port- 1 of UPFC with a fault reactance of 0.04 p.u. and cleared at 4.5 cycles.

The simulation results for case 1 with $V_{\mathrm{P}(\mathrm{se})}=0.065$ are shown in Figs. 15 and 16 for D-Q and three-phase model of UPFC, respectively. It is observed that, there is a good match between the simulation results of D-Q and three-phase model of UPFC. The shaft torque oscillations decay fast.

\subsection{Discussion}

Although, the damping torque method accurately predicts the torsional mode stability, the stability of the entire system can be studied by eigenvalue analysis. The introduction of UPFC in the transmission network, in general, has the ef-
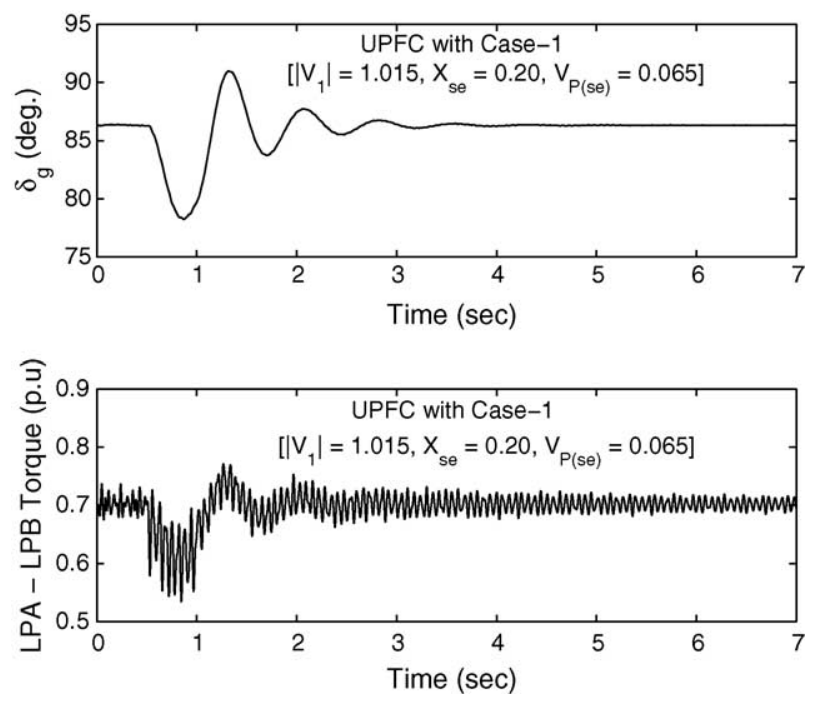

Fig. 14. Simulation with three-phase model of UPFC for step change in $T_{\mathrm{m}}$. 

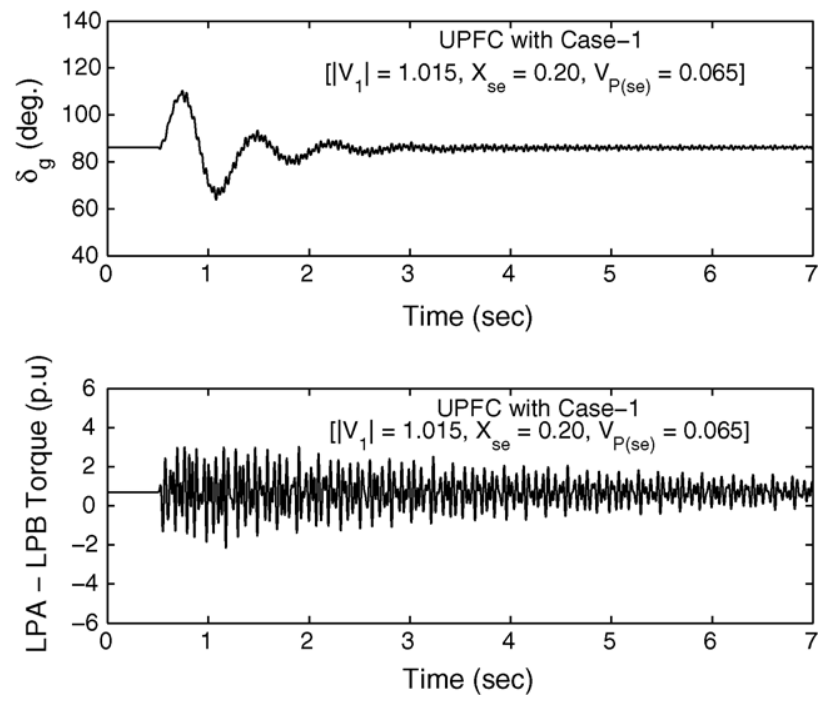

Fig. 15. Simulation with D-Q model of UPFC for three-phase fault.

fect of reducing the electrical resonance frequency due to the series reactive voltage injection and reducing the peak negative damping due to the series real voltage injection. This is shown in Fig. 17 where the damping torque with two operating modes of the UPFC are compared with the case without UPFC (where $60 \%$ series compensation is provided by fixed series capacitor alone).

Note that the resonant frequencies shown in Fig. 17 are the complements of the electrical resonant frequency.

The control of the real voltage plays an important role here. The 'resistance emulation' type of control is better than the fast control of port- 2 voltage of the UPFC (which is equivalent to the control of reactive power flow in the line). It is to be noted that, slow control of port- 2 voltage will have no significant effect on the torsional damping.
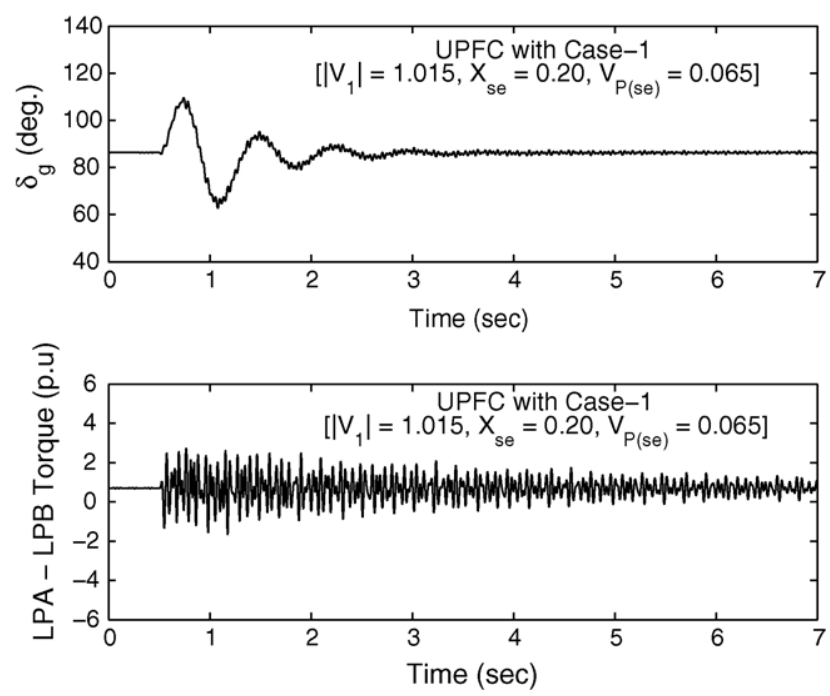

Fig. 16. Simulation with three-phase model of UPFC for three-phase fault.

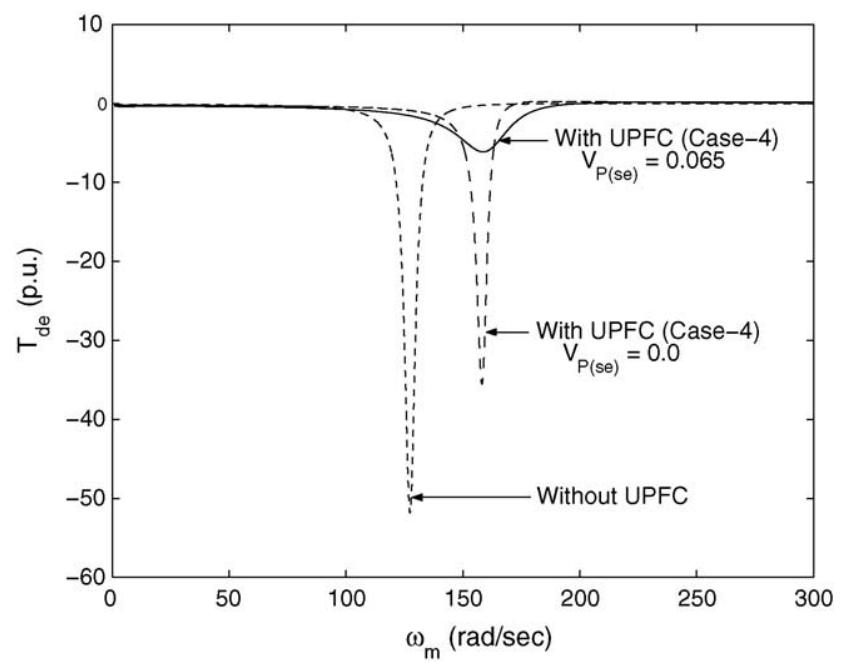

Fig. 17. Comparison of damping torque with and without UPFC.

The mode of control for the shunt VSC (constant reactive current or constant port-1 voltage) has little effect on the resonant frequency or the peak negative damping. However, the operating value of the reactive current has some effect on the torsional damping.

The eigenvalue analysis can be correlated with the damping torque analysis if it is assumed that the sensitivities of the real part of an eigenvalue corresponding to a torsional mode is constant. It can be shown that, the real part $\sigma_{i}^{1}$ and $\sigma_{i}^{2}$ for the $i$ th torsional mode for two different cases are approximately related by

$\Delta \sigma_{i}=\sigma_{i}^{1}-\sigma_{i}^{2}=\frac{T_{\mathrm{de} i}^{1}-T_{\mathrm{de} i}^{2}}{4 H_{\mathrm{m} i}}$

where $H_{\mathrm{m} i}$ is the modal inertia for the $i$ th mode, $T_{\mathrm{de} i}^{1}$ and $T_{\mathrm{de} i}^{2}$ are the damping torque coefficients for the two cases.

For the case study, from Tables 2 and $3, \Delta \sigma_{3}=\sigma_{3}^{1}-$ $\sigma_{3}^{2}=1.5185-0.8427=0.6758$. The R.H.S. of equation is calculated as 0.6701 at $159.3 \mathrm{rad} / \mathrm{s}$ (the torsional mode frequency with classical model of the generator). This shows the importance of damping torque analysis as a quick check on the torsional mode stability.

Although the damping torque analysis is approximate, it can be used as a fast screening tool. As shown in Appendix A, the computation of admittance function is speeded up as most of the network elements are passive and have simple expressions. The active elements (UPFC in this case) are represented individually by state space models and admittance functions are derived from them. The representation of the electrical system as a network simplifies the computation of the damping torque even for complex networks. This approach of computing the damping torque is novel. Although the IGE is ignored in the generator model, it can be approximately accounted by inserting a negative resistance in series with the generator stator. 
It is to be noted that, the active power control mode of UPFC is not considered here as it is not possible (in the IEEE FBM). The damping controllers also not considered as PSS is provided at the generator.

\section{Conclusions}

In this paper, we have presented the analysis and simulation of series compensated system with UPFC which is reported for the first time. The model of UPFC based on 12-pulse three-level VSCs is developed from the first principles taking in to consideration the switching action in three-phase VSC. For the linearized study which includes eigenvalue and damping torque analysis, the D-Q model is developed by neglecting the harmonics in the output voltage of VSCs. The method of damping torque analysis with UPFC is developed for the first time for the fast prediction of torsional mode stability. The application of the D$\mathrm{Q}$ model is validated by the transient simulation of threephase model of UPFC. The effectiveness of various combinations of operating modes of shunt and series converters in damping of SSR has been investigated. It is to be noted that while the techniques of eigenvalue analysis and simulation are directly applicable for multimachine systems with multiple controllers, the damping torque analysis needs to be slightly modified for application to multimachine systems.

The following points emerge based on the results of the case study.

(1) The operating mode of the shunt converter has no significant effect on the torsional modes.

(2) The injection of positive series real voltage improves the damping of the critical torsional mode. The resistance emulation mode is significantly better than the constant (port-2) voltage control (which is equivalent to the constant reactive power flow control).

(3) The injection of series reactive voltage for line compensation reduces the risk of SSR as not only the negative damping is reduced, the resonance frequency is increased. This indicates the possibility of detuning SSR by adjusting the series reactive voltage, when- ever feasible. In addition, a significant increase in the damping of torsional modes is achieved by emulating a positive resistance with the injection of series real voltage.

\section{Appendix A. Derivation of admittance function at the generator internal bus in D-Q axes}

The electrical system with UPFC shown in Fig. 1 can be represented as shown in Fig. A.1 for the purpose of damping torque analysis. In damping torque analysis, the generator is modelled by a constant voltage source $\left(E^{\prime}\right)$ behind a transient reactance $\left(X^{\prime}\right)$. The impedances of the network external to the generator (refer Fig. 1) are

$$
\begin{aligned}
& Z_{\mathrm{e} 1}(s)=R_{\mathrm{e} 1}+L_{\mathrm{e} 1} s+\frac{1}{C s} \\
& Z_{\mathrm{e} 2}(s)=R_{2}+L_{\mathrm{e} 2} s \\
& Z_{\mathrm{sh}}(s)=\frac{\omega_{\mathrm{B}}}{B_{\mathrm{C}} s}
\end{aligned}
$$

where

$$
\begin{aligned}
& R_{\mathrm{e} 1}=R_{1}, \quad L_{\mathrm{e} 1}=\frac{X^{\prime}+X_{\mathrm{t}}+X_{1}}{\omega B}, \\
& C=\frac{1}{\omega_{\mathrm{B}} X_{\mathrm{C}}}, \quad L_{\mathrm{e} 2}=\frac{x_{2}+X_{\mathrm{SYS}}}{\omega_{\mathrm{B}}}
\end{aligned}
$$

The expressions for impedance functions in D-Q axes for the passive elements are given in [1]. These are

$$
\begin{aligned}
Z_{i \mathrm{DD}}(s) & =Z_{i \mathrm{QQ}}(s)=\frac{1}{2}\left[Z_{i}\left(s+j \omega_{0}\right)+Z_{i}\left(s-j \omega_{0}\right)\right] \\
& =\Re\left[Z_{i}\left(s-j \omega_{0}\right)\right] \\
Z_{i \mathrm{DQ}}(s) & =-Z_{i \mathrm{QD}}(s)=\frac{j}{2}\left[Z_{i}\left(S-j \omega_{0}\right)-Z_{i}\left(s+j \omega_{0}\right)\right] \\
& =-\mathfrak{I}\left[Z_{i}\left(s-j \omega_{0}\right)\right]
\end{aligned}
$$

where $i=\mathrm{e} 1$, e2, sh.

To obtain the admittance function $\left[Y_{\mathrm{u}}\right]$ seen at port-1 of UPFC, in D-Q frame of reference, the UPFC equations (including the controllers) are linearized at the operating point

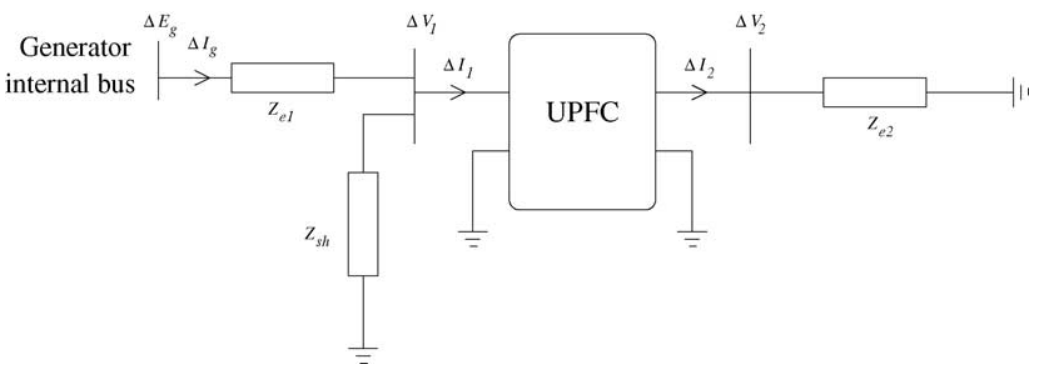

Fig. A.1. Linearized electrical system for damping torque calculations. 
and expressed as

$\left[\Delta \dot{X}_{\mathrm{u}}\right]=\left[A_{\mathrm{u}}\right]\left[\Delta X_{\mathrm{u}}\right]+\left[B_{\mathrm{u}}\right]\left[\begin{array}{c}\Delta V_{1} \\ \Delta I_{2}\end{array}\right]$

$\left[\begin{array}{c}\Delta I_{1 \mathrm{D}} \\ \Delta I_{1 \mathrm{Q}} \\ \Delta V_{2 \mathrm{D}} \\ \Delta V_{2 \mathrm{Q}}\end{array}\right]=\left[C_{\mathrm{u}}\right]\left[\Delta X_{\mathrm{u}}\right]+\left[D_{\mathrm{u}}\right]\left[\begin{array}{l}\Delta V_{1} \\ \Delta I_{2}\end{array}\right]$

where

$$
\begin{aligned}
& {\left[\Delta V_{1}\right]^{\mathrm{t}}=\left[\begin{array}{ll}
\Delta V_{1 \mathrm{D}} & \Delta V_{1 \mathrm{Q}}
\end{array}\right]} \\
& {\left[\Delta V_{2}\right]^{\mathrm{t}}=\left[\begin{array}{ll}
\Delta V_{2 \mathrm{D}} & \Delta V_{2 \mathrm{Q}}
\end{array}\right]}
\end{aligned}
$$

By taking Laplace transforms of Eqs. (A.6) and (A.7), we can express

$$
\left[\begin{array}{c}
\Delta I_{1} \\
\Delta V_{2}
\end{array}\right]=[H]\left[\begin{array}{c}
\Delta V_{1} \\
\Delta I_{2}
\end{array}\right]=\left[\begin{array}{ll}
h_{11} & h_{12} \\
h_{21} & h_{22}
\end{array}\right]\left[\begin{array}{c}
\Delta V_{1} \\
{\left[Y_{\mathrm{e} 2}\right] \Delta V_{2}}
\end{array}\right]
$$

where $\quad[H]=\left[\left[\mathrm{C}_{\mathrm{u}}\right]\left[s I-A_{\mathrm{u}}\right]^{-1}\left[B_{\mathrm{u}}\right]+\left[D_{\mathrm{u}}\right]\right]$ and $\left[Y_{\mathrm{e} 2}\right]=\left[\mathrm{Z}_{\mathrm{e} 2}\right]^{-1}$.

Eliminating $\Delta V_{2}$ from (A.8) and simplifying, we finally get the expression for the admittance matrix seen at the generator internal bus as

$[Y]=\left[\begin{array}{ll}Y_{\mathrm{DD}} & Y_{\mathrm{DQ}} \\ Y_{\mathrm{QD}} & Y_{\mathrm{QQ}}\end{array}\right]=\left[\left[Z_{\mathrm{e} 1}\right]+\left\{\left[Z_{\mathrm{Sh}}\right]^{-1}+\left[Y_{\mathrm{u}}\right]\right\}^{-1}\right]^{-1}$

where $\left[Y_{\mathrm{u}}\right]=\left\{\left[h_{11}\right]+\left[h_{12}\right]\left[Y_{\mathrm{e} 2}\right]\left[\left(I-h_{22} Y_{\mathrm{e} 2}\right)\right]^{-1}\left[h_{21}\right]\right\}$ and $I$ is identity matrix.

\section{References}

[1] K.R. Padiyar, Analysis of Sub-synchronous Resonance in Power Systems, Kluwer Academic Publishers, Boston, 1999.

[2] K.R. Padiyar, A.M. Kulkarni, Control design and simulation of unified power flow controller, IEEE Trans. Power Deliv. 13 (4) (1998) 1348-1354.

[3] K. Sen Kalyan, J. Stacy Eric, UPFC-unified power flow controller: theory, modelling and applications, IEEE Trans. Power Deliv. 13 (4) (1998) 1453-1460.

[4] Schauder, Mehta, Vector analysis and control of advanced static VAR compensators, IEE Proc. C 140 (4) (1993) 299-306.

[5] K.R. Padiyar, Nagesh Prabhu, Analysis of subsynchronous resonance with three-level 12-pulse VSC based SSSC, IEEE TENCON-2003 (14-17 October 2003) 76-80.

[6] N.G. Hingorani, L. Gyugyi, Understanding FACTS, IEEE Press, New York, 2000.

[7] R.W. Menzis, Y. Zhuang, Advanced static compensation using a multilevel GTO thyristor inverter, IEEE Trans. Power Deliv. 10 (2) (1995) 732-738.

[8] J.B. Ekanayake, N. Jenkins, Mathematical models of a three-level advanced static Var compensator, IEE Proc. Generat. Transm. Distrib. 144 (2) (1997) 201-206.

[9] K.R. Padiyar, Power System Dynamics-Stability and Control, second ed., B.S. Publications, Hyderabad, 2002.

[10] C.D. Schauder, et al., Operation of the unified power flow controller (UPFC) under practical constraints, IEEE Trans. Power Deliv. 13 (2) (1998) 630-639.

[11] B.A. Renz, et al., AEP unified power flow controller performance, IEEE Trans. Power Deliv. 14 (4) (1999) 1348-1354.

[12] I.M. Canay, A novel approach to the torsional interactions and electrical damping of the synchronous machine, part-I: theory, part-II: application to an arbitrary network, IEEE Trans. Power Apparatus Syst., vol. PAS-101, no. 10, 1982, pp. 3630-3640.

[13] K.R. Padiyar, R.K. Varma, Damping torque analysis of static Var system controllers, IEEE Trans. Power Syst. 6 (2) (1991) 458-465.

[14] The Math Works Inc., Using MATLAB-SIMULINK, 1999.

[15] IEEE Committee report, First benchmark model for computer simulation of subsynchronous resonance, IEEE Trans. Power Apparatus Syst., vol. PAS-96, no. 5, September-October 1977, pp. 1565-1572. 